\title{
A Bayesian model to estimate the cutoff and the clinical utility of a biomarker assay
}

\section{$1 \quad$ 1. Introduction}

3 The development of diagnostic tests using biomarkers is now an integral part of the drug discovery

4 and development process. Biomarkers are used in enrichment to assist in patient selection and in the

5 design of clinical trials [1]. In the field of oncology, for instance, biomarkers are used to develop tests

6 aiming to identify and treat those who are more likely to respond and demonstrate a higher therapeutic

7 benefit. The adaptation of these biomarkers based tests for classification purposes requires the

8 assessment of the test performance and, perhaps even more importantly, their clinical utility.

10 The evaluation of the diagnostic performance of a set of potential biomarkers is usually performed

11 using Receiver Operating Characteristic (ROC) curves, which plot the true positive rate (sensitivity)

12 versus the false positive rate (1-specificity) over all possible decision thresholds of the test. This is

13 helpful in choosing the most discriminating marker or set of markers [2]. After choosing an accurate

14 marker from a set of markers, an appropriate threshold, or cutoff value, must be determined such that

15 it correctly classifies patients as required.

16

17 Several strategies exist for selecting a cutoff value. These may be based on numerical results around

18 the sensitivity and specificity, but may also include criteria based on biological or physiological

19 information. Thus, optimal thresholds may vary depending on the underlying criteria [3]. Most

20 commonly, the optimal cutoff is chosen as the one that optimizes a utility function. For example, the

21 cutoff that maximizes the number of correctly classified patients or the cutoff that minimizes the 
22 misclassification cost. Because a utility function also requires information about cost or benefit, which

23 is not always available, the optimal cutoff value is found by using criteria related to ROC curves.

24 Confidence intervals around the cutoff value are obtained either using the delta method or, most

25 commonly, by employing bootstrapping, though the coverage probabilities can be far from the desired

26 level [4].

28 ROC-based methods, however, do not provide information on the diagnostic accuracy for a specific

29 patient. Particularly in situations where a diagnostic test is used for classification purposes, clinicians

30 are mainly concerned with the predictive ability of the test, approaching the result of the test from the

31 direction of the patients. The assessment of correct classifications can be facilitated by the use of

32 positive and negative predictive values (PPV and NPV, respectively). These predictive values are

33 functions of the accuracy of the test and the overall prevalence, and can be used to assess the clinical

34 utility of a diagnostic test for classification purposes.

36 Lunceford [5] discussed the estimation of the clinical utility of a biomarker assay in the context of

37 predictive enrichment studies. The aim of his research was to select a cutoff on a potentially predictive

38 biomarker that can be used as an enrollment criterion for patient selection. By implementing a

39 Bayesian approach in estimating clinical utility measures he facilitates cutoff decision making, but

40 without considering the actual cutoff estimation.

42 In this paper, we are interested in estimating the cutoff and the clinical utility of a biomarker, but most

43 importantly the uncertainty around the estimates of the parameters of interest. We propose a flexible

44 Bayesian approach that can utilize prior information to estimate the cutoff of a biomarker and its

45 credible interval. By modelling the probability of response with a step function using predictive 
46 values, we obtain estimates for the cutoff as well as for the predictive values of the test. Bayesian

47 analysis allows us to assign probability distributions to our prior beliefs for the parameters of interest

48 and combine these with the data likelihood to yield a posterior probability distribution representing our

49 updated belief.

51 In section 2, we present the Bayesian model for estimating the cutoff of a (continuous or ordinal)

52 biomarker for a binary outcome. The different prior specifications for the cutoff that we consider allow

53 for some robustness of the method. The finite-sample performance of the proposed Bayesian approach

54 is demonstrated through a series of simulations and compared with alternative frequentist methods like

55 Maximum Likelihood approach and the PSI index in Section 3. We also present applications of our

56 method in Section 4 on real data for a continuous biomarker and binary, as well as time-to-event

57 endpoints. Finally, we conclude with a brief discussion.

\section{2. Methods}

\section{$60 \quad 2.1$ Bayesian model for estimating the cutoff and its credible interval}

62 In this section we present a Bayesian model for estimating the posterior distribution of a cut-off value

63 for a biomarker, as well as its predictive values. Let $X=\left(X_{1}, X_{2}, \ldots, X_{n}\right) \in \mathbb{R}$ denote the continuous

64 biomarker measurements for $n$ individuals and assume that $X$ is available to be measured on all

65 patients. Let $Y=\left(Y_{1}, Y_{2}, \ldots, Y_{n}\right)$ denote the binary response variable, where $Y_{i} \in\{0,1\}$ for all $i=$

$661, \ldots, n$. is the response indicator (e.g. $Y_{i}=0$ denotes the non-responders and $Y_{i}=1$ the responder

67 subjects). We do not make assumptions about the distribution of the biomarker $X$ and by convention it 
68 will be assumed that high values of the marker $X$ are associated with increased probability of response

69 to a treatment.

71 We assume that the probability of response $p$ can be modeled by a step function (Figure 1), in terms of

72 positive predictive value (PPV) and negative predictive value (NPV) of the biomarker assay. The

73 Positive Predictive Value (PPV) is defined as the conditional probability of response given a positive

74 test result, i.e. $P\left(y=1 \mid T^{+}\right)$. Conventionally, for potential cutoff $c p \in \mathbb{R}$, the test is positive, $T^{+}$, if

75 the biomarker exceeds the cutoff, $X \geq c p$, and is negative otherwise. Similar statements apply for the

76 Negative Predictive Value (NPV) which is defined as the conditional probability that an individual is a

77 non-responder given a negative test result, i.e. $P\left(Y=0 \mid T^{-}\right)=P(Y=0 \mid X \leq c p)$. The model is

78 specified in the following way:

$$
Y \mid X \sim \operatorname{Bernoulli}(p)
$$

80

$$
p(x)=P(Y=1 \mid X=x)=\left\{\begin{array}{l}
p_{1}=P(Y=1 \mid X \leq c p), \text { for } x \leq c p \\
p_{2}=P(Y=1 \mid X>c p), \text { for } x>c p
\end{array}\right.
$$

82

83 The $p_{1}=1$ - NPV expresses the probability of response given $X$ is below the cutoff value $c p$ and

$84 p_{2}=\mathrm{PPV}$ expresses the probability of response given that $X$ is greater than $c p$.

85

86

87

88 Logistic regression can be used for decision making, i.e. to classify a subject as responder or not, only

89 in conjunction to a probability threshold, i.e. $p=0.5$ [6]. However, the advantage of using the step

90 function is that the cutoff is a parameter of the model and therefore a Bayesian approach can be

91 applied. The strong assumption we make that the probability of response can be modeled by a step 
92 function is probably not always reflecting the reality. However, it may serve as an approximating

93 model in cases where there are two populations that have a pronounced difference in the response rate.

94 It follows from literature on misspecified models [7], [8] that even, if the model is misspecified the

95 estimates of the assumed step function are consistent for the parameter values for which the assumed

96 model minimizes the distance from the true distribution in terms of Kullback-Leibler (KL) divergence

97 [9].

\section{$99 \quad$ 2.1.1 Prior specification}

101 In a Bayesian setup, the idea is to represent the uncertainty about the parameters by a prior

102 distribution. Prior information can take into account subjective beliefs about the values of the

103 parameters of the model. This external information can be historical information from experiments,

104 experts opinion or literature findings. A Bayesian approach can thus be useful as it allows flexibility

105 combining the available prior knowledge on test characteristics with new data. Importantly, incorrect

106 prior information can lead to unreliable posterior estimates, and therefore great attention should be

107 paid to the choice of the prior. On the other hand, if good prior information is available then the gain is

108 in the precision of the estimates.

109

110 Here, the parameters $p_{1}, p_{2}$ and the cutoff are assumed to have probability distributions reflecting the

111 uncertainty in their parameters values. For the probabilities of response $p_{1}$ and $p_{2}$, we consider

112 distributions that the support set is the interval $(0,1)$. Furthermore, we require that $p_{2}>p_{1}$. The

113 simplest case is to assign uniform priors, i.e.

$$
p_{1} \sim \operatorname{Unif}(0,1) \quad \text { and } \quad p_{2} \sim \operatorname{Unif}\left(p_{1}, 1\right)
$$

115 Other options may include Truncated Normal or Beta distributions. 
117 For the cutoff $c p$, we can consider an informative prior, if prior information is relevant and an

118 uninformative prior, when there is no information available, usually expressed by a uniform

119 distribution. Finally a weighted sum of informative and non-informative priors can be considered to

120 acknowledge potential prior-data conflict. We propose here a two-component mixture of priors, which

121 allow for robustness. The first component of the mixture prior is the informative part which expresses

122 the subjective belief we have and is derived from prior experiments, animal data or literature. Then

123 second component, is the weakly (or non-) informative part that ensures robustness against potential

124 prior-data conflict. We characterize a prior distribution as weakly informative if the information that

125 provides is intentionally weaker than whatever actual prior knowledge is available.

127 As discussed by Schmidli et.al [10], since one of the mixture components is usually vague, mixture

128 priors will often be heavy tailed and therefore robust. Let $g_{1}$ be the probability density function (pdf)

129 of the uninformative component and $g_{2}$ the pdf for the informative part. The mixture prior can be

130 expressed as:

$c p=w g_{1}+(1-w) g_{2}$

132 with $\quad w \sim \operatorname{Beta}(1,1)$

133 The weight parameter $w$ will be updated at each iteration by the Bayesian model as described in 134 section 3.

\section{2.1.2. Prior specification for constrained positive predictive value}

138 In this section, we present the case where the objective is to estimate a cutoff associated with a 139 targeted clinical utility value by controlling the PPV of the test. For example, we might be interested 
140 in the posterior distribution of the cutoff expected to yield a PPV between $70 \%$ and $100 \%$ or a $1-\mathrm{NPV}$

141 to be between 0 and $20 \%$. Whether a cutoff that yields a pre-specified predicted value exists would of

142 course depend on the relationship between the biomarker and the response. The idea is then to

143 incorporate the restriction on the predictive values via the prior information and require that only

144 information on the pre-specified domain are acceptable. In that case, the constraints can be controlled

145 through priors, e.g.

$146 \quad p_{1} \sim \operatorname{Unif}\left(0, p_{2}\right) \quad$ and $\quad p_{2} \sim \operatorname{Unif}(0.7,1)$

147 It is worth noting that even if the parameter is constrained such that the actual desired range is not

148 achievable e.g. $p_{2} \notin(0.7,1)$, the method will result in the cut-point that is as close as possible to

149 achieve this constraint (i.e. the mass of the posterior density is on the lower bound of the constrained

150 interval)

151

\section{2.1.3. Posterior distribution}

153

154 The posterior distribution of interest is formulated as

$155 \quad f\left(c p, p_{1}, p_{2} \mid x, y\right) \propto L\left(p_{1}, p_{2}, c p \mid x, y\right) \times f\left(p_{1}\right) \times f\left(p_{2}\right) \times f(c p)$

156 where $L\left(p_{1}, p_{2}, c p \mid x, y\right)$ is the likelihood function of the data and $f(\cdot)$ denotes the density of the prior

157 and $f(\cdot \mid x, y)$ the posterior density of the distribution of the parameters.

\subsubsection{Maximum Likelihood Estimation}

161 The log likelihood of the model described in section 2.1 is given by

$$
\log L=L\left(p_{1}, p_{2}, c p \mid x, y\right)=\sum_{i=1}^{n} y_{i} \log (p)+\left(1-y_{i}\right) \log (1-p)
$$

165 with $p$ as stated in (2.1) and $n$ denotes the total sample size. The log likelihood function becomes 


$$
\log L=\sum_{i=1}^{n_{1}} y_{i} \log \left(p_{1}\right)+\left(1-y_{i}\right) \log \left(1-p_{1}\right)+\sum_{i=1}^{n_{2}} y_{i} \log \left(p_{2}\right)+\left(1-y_{i}\right) \log \left(1-p_{2}\right)
$$

167 Where $n_{1}, n_{2}$ denote the sample size for the population that has $X \leq c p$ and $X>c p$ respectively.

168 The maximum likelihood estimates $\widehat{c p}, \widehat{p_{1}}$ and $\widehat{p_{2}}$ are obtained by first minimizing - $\log L$ with respect

169 to $p_{1}$ and $p_{2}$, for given $c p$ and then maximizing the resulting profile likelihood with respect to $c p$. One

170 can see that $\widehat{p_{1}}$ and $\widehat{p_{2}}$ are just the average response rates in the subsamples $\left\{x_{i} \leq \widehat{c p}\right\}$ and $\left\{x_{i}>\widehat{c p}\right\}$

171 where $x_{i}$ are the observed values of $X$ (see the appendix for a similar argument for the population

172 parameters).

173

\section{3. Simulation Study}

176 In this section we examine the bias of the estimated cutoff under different distributional assumptions

177 for the biomarker $X$ via simulations. We compared the proposed Bayesian method with two frequentist

178 approaches; the Maximum Likelihood Estimator (MLE) and the Predictive Summary Index (PSI) [11].

179 The PSI estimates the optimal cutoff by maximizing the difference in predictive values for all possible

180 cutoffs $c$ and is expressed as $P S I=\max _{c}\{P P V(c)+N P V(c)-1\}$. The PSI is derived in the target

181 (patient) population as a measure of the goodness of the predictability in a diagnostic test, thus, is a

182 more comprehensive measure than the Youden index [12] in a clinical setting. For the latter approach,

183 the confidence intervals are calculated by the bootstrap method by resampling the data $B=500$ times,

184 calculating the $\widehat{P S I}_{J}$ per sample $j=1, \ldots, B$. and then taking $\alpha / 2$ and $1-\alpha / 2$ quantiles of the $\widehat{P S I}_{J}$ to

185 construct a $(1-\alpha) 100 \%$ CI. For the Bayesian approach, the credible intervals are obtained by using

186 the empirical $\alpha / 2$ and $1-\alpha / 2$ quantiles of the posterior distribution (quantile method). A level of

$187 \alpha=0.05$ was used for both methods. 
189 We include in our results the Maximum Likelihood Estimator (MLE) of the parameters $p_{1}, p_{2}, c p$

190 together with the 95\% Confidence Intervals (CI) as a comparison. In general, maximum likelihood

191 methods do not perform well when parameter estimates are on the boundary of the parameter space

192 [13], leading to some non-convergence issues. On the other hand, Bayesian inference via MCMC

193 algorithms permits full posterior inference even in the absence of asymptotic normality [14] and have

194 no issues with parameter estimates on the boundary. In our simulation we did not anticipate any

195 optimization issues regarding the optimization with the ML method.

197 We simulated 10000 datasets on which we applied all methods. We also report the coverage

198 probability and the width of the credible and confidence intervals over the simulation runs. The

199 analysis for the MLE and PSI estimation was done in R version 3.3.3 [15]. The 10000 datasets were

200 generated in R (for the MLE and PSI estimation) and then exported to SAS version 9.4 (SAS Institute

201 Inc., Cary, NC, USA) (for the Bayesian estimation), such that the analysis was consistent for all the

202 methods. For the PSI method the R-package "OptimalCutpoints" [16] was used and for the profile

203 MLE the R-library "bbmle” [17].

205 The posterior computation was done by using Markov Chain Monte Carlo (MCMC). In our analysis

206 we used the Metropolis-Hastings [18], [19] iterative sampling method to approximate the posterior

207 distribution and get posterior estimates for the parameters in (2.4). Posterior computation was

208 conducted using PROC MCMC procedure in SAS. The burn-in consisted of 10000 iterations, and 50

209000 subsequent iterations were used for posterior summaries. Convergence of the MCMC chain was

210 checked for randomly selected number of iterations, using diagnostic plots and the Gelman-Rubin

211 convergence statistic as well as visually via trace plots, sample autocorrelations and kernel density

212 plots. The SAS and R code can be found in the appendix. 


\section{$214 \quad 3.1$ Simulation Setting}

\section{3.1.1 Generating data using a step function and a logistic function}

217 The true model that was used to generate the binary outcome $y$ has one biomarker $X$. We consider six

218 different simulation scenarios, each with $n=200$, and $n=50$. Furthermore, we assumed that the

219 biomarker $X$ follows different distributions as shown in Table 1. Each component of the response

220 vector $y$ is viewed as a realization of a Bernoulli random variable with probability of success $p$, i.e.

$221 y \mid X \sim \operatorname{Bermoulli}(p)$. In scenarios 1-4 and 6 the generating model has response probability $p$

222 expressed as a step function, with $p(X)=\left\{\begin{array}{ll}p_{1}, & \text { if } X \leq c p \\ p_{2}, & \text { if } X>c p\end{array}\right.$, whereas in scenario 5 the generating

223 model is a logistic model with probability of response $p=\frac{e^{X \beta}}{1+e^{X \beta}}$.

225 The primary purpose of including scenario 5 is to investigate the behavior of the Bayesian method

226 (together with the MLE and the PSI method), when the fitted model is divergent from the true

227 underlying model. For this scenario, the true $c p, p_{1}$ and $p_{2}$ are not defined by the data generating

228 mechanism. In fact, it is known (see e.g. [7],[8]) that the estimated parameters from the Bayesian and

229 MLE method, are consistent for the ones that minimize the Kullback-Leibler divergence between the

230 fitted (step) model and the true (logistic) model. We give details on the limiting population parameter

231 in the Appendix.

233 In scenario 4, we explore the case that the biomarker $X$ is ordinal. The data were generated in the

234 following way; Assuming $X \sim \operatorname{Normal}\left(\mu=7, \sigma^{2}=1\right)$ as in scenario 1, we calculate the quartiles of $X$

235 that form the four levels of the ordinal variable (the lowest quartile corresponds to category $X=1$ and 
236 the $4^{\text {th }}$ quartile to $X=4$ ). Each component of the response $Y$ is a realization from a Bernoulli random

237 variable with $p(X)=\left\{\begin{array}{c}p_{1}, \text { if } X=1,2 \\ p_{2}, \text { if } X \geq 3\end{array}\right.$.

239 Moreover, we are interested to address the case that the true generating model has two cutoffs and the

240 fitted model assumes only one cutoff (scenario 6 in Table 1). To simulate data for this scenario,

241 scenario 6, we assumed that $p(X)=\left\{\begin{array}{lc}p_{1}, & \text { if } X \leq c p_{1} \\ p_{2}, & \text { if } c p_{1}<X \leq c p_{2} . \text { If the data indicate the existence of } \\ p_{3}, & \text { if } X>c p_{2}\end{array}\right.$

242 two cut-off values, this might indicate the existence of two subgroups with different response

243 probabilities. For the scenarios 2 and 6, we assumed that the biomarker $X$ follows a mixture of two

244 normal distributions expressed as $X \sim \operatorname{Normal}\left(\mu=\mu_{1}, \sigma^{2}=\sigma^{2}{ }_{1}\right)+\operatorname{Normal}\left(\mu=\mu_{2}, \sigma^{2}=\sigma^{2}{ }_{2}\right)$.

[Table 1 about here]

\subsection{Simulation Results}

250 This section describes the simulation results regarding the finite sample properties of the estimators

251 from the Bayesian method, the PSI index and the ML. In our results, we chose to report the Bayesian

252 posterior mean, as we consider it an adequate measure to summarize the posterior density and we

253 found that the cutoffs were generally similar whatever estimate kept from the posterior distribution

254 among the mode, median or mean. In Table 2 and Table 3 we report the Bias of estimators for $c p$

255 (Table 2), $p_{1}, p_{2}$ (Table 3) for scenarios 1-4 based on 10000 simulation runs. Coverage probability

256 and interval width of the confidence and credible intervals are shown in Table 4 and Table 5. 
258 For the Bayesian method, we also report results for four different prior specifications. The first, the

259 naïve case, corresponds to a uniform prior (UP) in the interval of the range of the biomarker

260 measurements. Note here that with a uniform prior, it is well known [20] that, the Bayesian posterior

261 mode corresponds to the ML estimator. Other priors we considered are a perfect informative prior

262 (denoted as IPP), an imperfect informative prior (denoted as IPN) and two mixture priors (MixP and

263 MixN) each with two components; a weighted sum of a uniform and informative prior (UP+IPP) and a

264 uniform and imperfect informative prior (UP+IPN) respectively. More specifically, for the IPP prior,

265 we assume a distribution for which the true cutoff lies in an interval of high probability, whereas for

266 the IPN prior the true cutoff lies in one of the tails of the distribution. An illustration of the IPP and

267 IPN priors used for scenario 1 can be found in Figure 2. Obviously, when the prior does not include

268 the true value of the cutoff, then the posterior estimates are expected to be biased for finite sample

269 sizes. The priors for $p_{1}, p_{2}$ were taken as uniform distributions as given by (2.2).

[Figure 2 about here]

273 Regarding the estimation of the cutoff $c p$, in scenarios 1-4, results in Table 2 show that estimators

274 using all three methods behave similarly in terms of bias, resulting in nearly unbiased estimators. The

275 Bayesian method gives a much better coverage than the MLE and PSI methods for the scenarios where 276 the marker is continuous (Table 4). For the PSI method in scenarios 1 and 3, the bias of the estimate of

$277 c p$ is far too high in absolute terms (see Table 2). Additionally, the coverage of the bootstrapped

278 confidence interval is far from the nominal level and the interval width is much wider compared to the

279 other methods. The Bayesian method performs either the same or better compared to MLE and PSI in 280 terms of bias and coverage both in case of the continuous and the ordinal biomarker. 
282 For all priors that we considered, the resulting estimators are on average unbiased for both $n=200$

283 and $n=50$. As expected, with the robust mixture prior and the informative prior, estimates have the

284 smallest bias on average. The IPP prior gives a smaller interval width with the mixture prior second.

285 Moreover, with the IPP prior we get more precise estimates while obtaining the same or better

286 coverage compared to the other prior specifications.

\section{[Table 2 about here]}

\section{[Table 3 about here]}

To see how the prior affects the estimation, we calculate the absolute difference between the estimated

292 and true value of the cutoff over the simulation runs and we present the results for the Bayesian

293 method for scenario 1 for all different prior specifications as shown in Figure A.1 in the Appendix. In

294 Figure A.1, we see that the absolute difference between the estimate and the true value of $c p$ was on

295 average below 10\%. As for the predictive values, we discuss our findings for $n=200$ and show the

296 results for the estimate of the cutoff. Detailed figures for the predictive values for $n=50$ can be found

297 in Table A.1 and Table A.2 in the Appendix.

299 As shown in Table 3 and Table 5, all methods performed well with good coverage and very small bias

300 for both $p_{1}$ and $p_{2}$. The bias of the estimates for the predictive values $p_{1}$ and $p_{2}$, was always below $1 \%$

301 for all scenarios. Coverage probabilities for the credible intervals reach the nominal value for the

302 Bayesian and the ML method but is not always the case for the estimation of $p_{2}$ when using the PSI

303 index as seen, for example, in scenario 1 and scenario 3, where the coverage probability for the PSI 
304 method is far from the nominal (Table 5). The length of the credible interval (for the Bayesian

305 method) was similar to the confidence interval for the MLE and always narrower compared to PSI.

306

307

308

309

310 For scenario 5 where the true model is generated assuming a logistic response curve, we estimated the

311 cutoff and the corresponding probabilities of response by applying the Bayesian method as well as the

312 MLE and the PSI approaches. In that case, the true cutoff is not directly defined by the data generating

313 mechanism. However, the population parameters are defined by minimizing the KL divergence

314 between the true (logistic) and the assumed (step) model as discussed in section 2.1 and more detailed

315 in the Appendix. The results of the distribution of the estimates of the parameters for scenario 5 for the 316 three methods are shown in boxplots in Figure 3.

317

318 In this scenario, the Bayesian estimates are more consistent and have a smaller variability compared to 319 the MLE and the PSI method. As can be seen from the boxplots, the ML and the PSI methods result in 320 heavy tailed distributions for all the parameters and especially for the estimate of the cutoff. The

321 estimates concerning the cutoff and the predicted values obtained with the PSI method, differ

322 significantly as compared to the other two methods. This is partially due to the fact that the PSI

323 optimizes a different utility function than the Bayesian and the ML approach. While the Bayesian and

324 the ML methods use the likelihood as an objective function, the PSI method seeks to maximize the

325 difference between predictive values (PPV- (1-NPV)) 
329 For scenario 6, the generating model assumes that there exist two cutoff values and three response 330 probabilities $p_{1}, p_{2}, p_{3}$ respectively. The Bayesian model we fit to estimate the cutoff and the 331 corresponding predictive values, assumes that there is only one cutoff value. For simplicity we used an

332 UP prior for the Bayesian method. The results of the fitted model are shown in Figure 4. Focusing on

333 the estimate of $c p$, we analyzed the results in more detail. We checked whether the obtained posterior

334 distribution was bimodal, and if so, we reported the two modes. To check for bimodality, i.e. if the

335 posterior density function has two peaks, we used the Hartigan's dip test for unimodality [21]. A p-

336 value less than 0.05 is taken to indicate non-unimodality (it means at least bimodality).

[Figure 4 about here]

340 Figure 5 shows the distribution of the estimated cutoffs when posterior density is judged to be

341 unimodal (5 733 out of 10000 simulations) and when it is found to be a bimodal posterior distribution

342 (4 267 out of 10000 simulations). Looking across all simulations we see that the cutoff is somewhere

343 between the two true cutoffs. When only a single mode is identified there is a clear tendency to be

344 close to the second true cutoff $c p_{2}=10$. When two modes are found, the underlying two true cutoffs 345 are estimated reasonably well despite the model misspecification. 


\subsection{The prostate cancer data}

353 We consider the prostate specific antigen (PSA) study of 12000 men aged 50-65, which was a

354 randomized study with a beta-carotene group as the treatment group vs. a placebo group. A substudy

355 reported by Etzioni et al. [22] analyzed serum levels of total PSA (on the log scale) for 683 subjects.

356 The dataset is described in [2] and [23] where you can find additional details about the study, which

357 was analyzed from a non-Bayesian perspective. The primary scientific question under investigation

358 was whether PSA could be used to diagnose prostate cancer, and was found that the total PSA is a

359 significant predictor of the occurrence of cancer with fairly good accuracy. Albeit the good diagnostic

360 ability of the marker PSA, we are interested in estimating a cutoff that takes into account the clinical

361 benefit of this marker.

362

363 In this paper, we considered response to a treatment as the outcome of interest but the method can be

364 used also when we refer to diagnostic tests, where the outcome is presence of disease or not. We

365 analyzed the data described above by applying our Bayesian method to estimate the cutoff related with

366 disease rates. Probabilistic statements are derived for the optimal cutoff as well as the predictive

367 values of the marker $(\operatorname{logPSA})$. We assume a uniform prior for the cutoff in the interval $(0,100)$ and

368 priors for the predictive values defined as in (2.2). We also report the ML estimator and the PSI index.

370 Figure 6 shows the posterior distributions for the cutoff (left panel) and the predictive values $p_{1}$ and $p_{2}$

371 (middle and right panels respectively). The MLE of the cutoff was found equal to 3.65 with 95\% CI

372 (3.62-3.69), while the posterior median was 3.66 with 95\% credible interval (2.44-3.95). The PSI

373 index which, we remind that maximizes a different objective function, estimates the optimal cutoff to

374 be 37.66 with $95 \%$ bootstrapped CI (7.90-43.30). At that cut-off the PPV and 1-NPV was equal to 1 
375 and 0.32 respectively. The Bayesian posterior mean for $p_{1}$ and $p_{2}$ were found equal to 0.17 with $95 \%$

376 credible interval (0.13-0.22) and 0.73 with $95 \%$ credible interval (0.61-0.79) respectively. The MLE

377 for $p_{1}$ was 0.18 with $95 \%$ confidence interval $(0.15-0.21)$ and for $p_{2}$ was 0.75 with $95 \%$ confidence

378 interval (0.68-0.81).

379

[Figure 6 about here]

381

382

\subsection{Application on survival data: Weibull model for melanoma data}

384 To illustrate that the proposed approach is useful for more complex settings we consider identifying

385 the appropriate cutoff for a time to event endpoint. For the following applications on time to event 386 data, we assume the following let $T_{i}$ denote the event time for subject $i$. Due to censoring, instead of

387 observing $T_{i}$, we observe the bivariate vector $\left(\min \left(T_{i}, C_{i}\right), \Delta_{i}\right)$ where $\Delta_{i}=I\left(T_{i} \leq C_{i}\right)$ with $I$ the 388 indicator function and $C_{i}$ is the censoring time.

390 The data used are the melanoma dataset available from the R package timereg [24]. The data consist

391 of measurements made on patients with malignant melanoma and patients with a thick tumor are

392 thought to have an increased chance of death from melanoma, thus the objective is to estimate a cut-

393 off value on (the log scale of) the tumor size such that the patients below and above the cutoff have a

394 pronounced difference in their hazard rates. We run the analysis using the R package MHadaptive [25]

395 and we used uniform priors for all the parameters. The R-code is available upon request from the 396 author. 
398 To set up the model in the survival setting, the thickness of the tumor on the log scale is denoted by $X$,

$399 T$ denotes time to death and is assumed to have a Weibull distribution with shape parameter $r$ and

400 scale parameter $\lambda$. The assumption is that, based on the thickness of the tumor, we can estimate a

401 cutoff $c p$ such that the two groups defined by $c p$, have different hazard functions. Therefore, the shape

402 and scale parameter for the patients that thickness of their tumor is below $c p$ is $r_{1}$ and $\lambda_{1}$ respectively

403 and accordingly, $r_{2}$ and $\lambda_{2}$ for those patients with $X>c p$.

404

$$
T \mid X \sim \text { Weibull }(r, \lambda) \quad \text { with } r=\left\{\begin{array}{ll}
r_{1}, & \text { if } X \leq c p \\
r_{2}, & \text { if } X>c p
\end{array} \text { and } \lambda= \begin{cases}\lambda_{1}, & \text { if } X \leq c p \\
\lambda_{2}, & \text { if } X>c p\end{cases}\right.
$$

405

406 Figure 7 (A) shows the posterior densities for the cutoff, the shape and scale parameters. We took the 407 medians of the posterior densities as point estimates for each parameter. In Figure 7 (B) we plot the

408 survival curves, estimated with the Kaplan-Meier estimate, for the patients bellow and above the

409 posterior cutoff estimate, which was taken as the posterior mean equal to $\widehat{c p}=5.38$ with $95 \%$

410 credible interval (5.07-5.86). At the same figure we plot the survival curves for the Weibull model in

411 dashed lines. As seen from the plot, the survival probability decreases with higher tumor thickness

412 value. To test whether the survival curves for the patients below and above the estimated cutoff value

413 differ significantly, we applied the log-rank test which showed that there is a significant difference in

414 survival $(\mathrm{p}<<0.05)$. Figure 7 (C) shows the hazard function for the two groups by plugging in the

415 estimated shape and scale parameters, i.e. the hazard function for the Weibull model becomes $h(t)=$

$416\left\{\begin{array}{l}\frac{r_{1}}{\lambda_{1}}\left(\frac{t}{\lambda_{1}}\right)^{r_{1}-1}, \text { if } X \leq c p \\ \frac{r_{2}}{\lambda_{2}}\left(\frac{t}{\lambda_{2}}\right)^{r_{2}-1}, \text { if } X>c p\end{array}\right.$, with $r_{1}, \lambda_{1}, r_{2}, \lambda_{2}$ taken as the means of the posterior densities.

$418 \quad$ [Figure 7 about here] 


\section{Discussion}

420 To enable targeted therapies and enhance medical decision making, biomarkers are increasingly used

421 in diagnostic tests. When using quantitative biomarkers for classification purposes, defining a reliable

422 cutoff value for the biomarker is a critical step in the drug development process, as the patient

423 selection process in the subsequent development steps may depend on this value. Although

424 classification probabilities, sensitivity and specificity, are considered more relevant to quantify the

425 inherent accuracy of the test, predictive values quantify the clinical utility of the test.

427 We have proposed a Bayesian method to estimate the cutoff value of a biomarker assay using the 428 predictive values, and also determine the uncertainty around these estimates. We used a step function,

429 which serves as an approximate model facilitating classification into two groups that have a

430 pronounced difference in their response rates. The advantage of using the step function is that the

431 cutoff and predictive values are parameters of the model. Even in the case that the assumption of a step

432 function is strong and the model is misspecified, the estimates of the assumed step function are

433 consistent for the parameter values for which the assumed model minimizes the distance from the true

434 distribution in terms of Kullback-Leibler divergence [7], [8]. A more careful investigation of this 435 approach is worth further exploration.

437 As mentioned by a referee, one could alternatively use a standard classification algorithm, like for 438 example logistic regression with a probability threshold of $p=0.5$. One could also choose $p$ such that 439 the Brier score [26], a measure of accuracy of predictions, is minimized. These methods do not

440 directly address the goal of population separation with regard to positive and negative predictive 441 values. Moreover, they do not directly provide credible or confidence intervals for the parameters of 
442 interest which was one of the major goals of the proposed method. Nevertheless, we have compared

443 the Bayesian approach with these methods and found that the estimated parameters of $c p$ are more

444 biased compared to the Bayesian estimates. Detailed figures can be found in the Appendix.

446 The proposed Bayesian approach allows for the estimation of the distribution of the cutoff for

447 continuous and ordinal biomarkers and permits probabilistic statements about the cutoff values and,

448 say, the response rates in the two groups. Together with the potential incorporation of prior

449 information, this is deemed useful especially in the earlier phases of drug development. Results

450 suggest that the proposed Bayesian method is very tractable in estimating the parameters of interest,

451 resulting in point estimators (e.g. posterior mean) that are practically unbiased in all scenarios, for all

452 prior constellations and sample size assumptions.

454 In this article, we presented four different prior specifications, including uninformative, informative, 455 and mixture priors. In all cases, estimation gave satisfying results. Especially when more accurate 456 prior information is available, the estimated parameters are nearly unbiased with high precision and

457 good coverage. We suggest a mixture prior that works well in practice, as it is robust towards potential 458 prior-data conflict. For a dataset of $n=200$ observations, the Bayesian approach takes 6.3sec to run 459 on a windows machine with processor Intel Xeon CPU E7-8867 v3 @ 2.5GHz, compared to 460 frequentist approaches (MLE 0.15sec and for PSI 3.7sec together with the bootstrapped CI). Although 461 the computational time for the proposed approach is increased, as is the case for Bayesian methods, is 462 not prohibitive.

464 The approach described in this article can be used as a basis for further investigation. The suggested 465 method was applied to a single biological marker, but it can be generalized to multiple markers. One 
way to deal with multiple markers is to estimate a composite score for each patient using a

467 combination of markers (under some working model, for example, under the logistic model), and then

468 consider this score as the new marker. Furthermore, it would be of great interest to consider the

469 generalization of the method to estimate multiple cutoffs that can be used potentially for subgroup

470 identification. In that case, model selection can be used to decide how many cut-offs (indicating the

471 number of subgroups) the model can have according to the data.

\section{Declaration of Conflicting Interests}

The Authors declare that there is no conflict of interest.

\section{References}

1. Colburn WA. Biomarkers in drug discovery and development: from target identification through drug marketing. The Journal of Clinical Pharmacology. 2003 Apr 1;43(4):329-41

2. Pepe MS. The statistical evaluation of medical tests for classification and prediction. Medicine; 2003.

3. Perkins NJ, Schisterman EF. The inconsistency of "optimal" cutpoints obtained using two criteria based on the receiver operating characteristic curve. American journal of epidemiology. 2006 Jan $12 ; 163(7): 670-5$.

4. Schisterman EF, Perkins N. Confidence intervals for the Youden index and corresponding optimal cut-point. Communications in Statistics Part B: Simulation and Computation. 2007 May;36(3):549563

5. Lunceford JK. Clinical utility estimation for assay cutoffs in early phase oncology enrichment trials. Pharmaceutical statistics. 2015 May 1;14(3):233-341.

6. Lever, J., Krzywinski, M. and Altman, N., 2016. Points of significance: Logistic regression

7. Huber PJ. The behavior of maximum likelihood estimates under nonstandard conditions. In Proceedings of the fifth Berkeley symposium on mathematical statistics and probability 1967 Jun (Vol. 1, No. 1, pp. 221-233).

8. Bunke O, Milhaud X. Asymptotic behavior of Bayes estimates under possibly incorrect models. The Annals of Statistics. 1998;26(2):617-44. 
9. Kullback S, Leibler RA. On information and sufficiency. The annals of mathematical statistics. 1951 Mar 1;22(1):79-86.

10. Schmidli H, Gsteiger S, Roychoudhury S, O'Hagan A, Spiegelhalter D, Neuenschwander B. Robust meta-analytic-predictive priors in clinical trials with historical control information. Biometrics. 2014 Dec 1;70(4):1023-32.

11. Linn S, Grunau PD. New patient-oriented summary measure of net total gain in certainty for dichotomous diagnostic tests. Epidemiologic Perspectives \& Innovations. 2006 Dec;3(1):11.

12. Youden WJ. Index for rating diagnostic tests. Cancer. 1950 Jan 1;3(1):32-5.

13. Chu H, Cole SR. Estimation of risk ratios in cohort studies with common outcomes: a Bayesian approach. Epidemiology. 2010 Nov 1;21(6):855-62.

14. Wagenmakers EJ, Lee M, Lodewyckx T, Iverson GJ. Bayesian versus frequentist inference. InBayesian evaluation of informative hypotheses 2008 (pp. 181-207). Springer, New York, NY.

15. Team RC. R: a language and environment for statistical computing. R Foundation for Statistical Computing. R version 3.3.3 Vienna, Austria.

16. Lopez-Raton M, Rodriguez-Alvarez MX, Cadarso-Suarez C, et al. Optimal cutpoints: An R package for selecting optimal cut-points in diagnostic tests. J Stat Software 2014; 61: 1-35, http//www.jstatsoft.org

17. Bolker B. R Development Core Team. 2014. bbmle: Tools for general maximum likelihood estimation. R package version 1.0. 17. Computer program. 2011.

18. Hastings WK. Monte Carlo sampling methods using Markov chains and their applications. Biometrika. 1970 Apr;57(1):97-109.

19. Metropolis N, Rosenbluth AW, Rosenbluth MN, Teller AH, Teller E. Equation of state calculations by fast computing machines. The journal of chemical physics. 1953 Jun;21(6):1087-92

20. Ibrahim JG, Chen MH, Sinha D. Bayesian survival analysis. John Wiley \& Sons, Ltd; 2005 Jul.

21. Hartigan JA, Hartigan PM. The dip test of unimodality. The annals of Statistics. 1985 Mar 1:7084.

22. Etzioni R, Pepe M, Longton G, Hu C, Goodman G. Incorporating the time dimension in receiver operating characteristic curves: a case study of prostate cancer. Medical Decision Making. 1999 Aug;19(3):242-51.

23. Broemeling LD. Advanced Bayesian methods for medical test accuracy. CRC Press; 2016 Apr 19. 
24. Martinussen T, Scheike T. Dynamic regression models for survival analysis. Statistics for biology and health. Springer. 2006

25. Chivers C. MHadaptive: general Markov Chain Monte Carlo for Bayesian inference using adaptive Metropolis-Hastings sampling.

26. Brier, G., 1950. Verification of forecasts expressed in terms of probability. Mon Wea Rev., pp. $78 ; 1-3$. 